\title{
Finite Element Methods for Elliptic Equations Using Nonconforming Elements
}

\author{
By Garth A. Baker
}

\begin{abstract}
A finite element method is developed for approximating the solution of the Dirichlet problem for the biharmonic operator, as a canonical example of a higher order elliptic boundary value problem.

The solution is approximated by special choices of classes of discontinuous functions, piecewise polynomial functions, by virtue of a special variational formulation of the boundary value problem. The approximating functions are not required to satisfy the prescribed boundary conditions.

Optimal error estimates are derived in Sobolev spaces.
\end{abstract}

1. Introduction. In this work a finite element method is proposed for approximating the solution of the Dirichlet problem for elliptic equations.

Although the method is applicable to general $2 m$ th order operators with inhomogeneous boundary condition, for reasons of clarity and ease of exposition, the method is displayed here on a simple 4th order case, the Dirichlet problem for the biharmonic operator with homogeneous boundary conditions.

The main features of this method lie in the fact that it uses nonconforming elements, optimal error estimates are derivable in the space $L^{2}$, and the piecewise polynomial functions used to approximate the solution are not required to satisfy the prescribed boundary conditions.

There are existing finite element methods treating higher order elliptic equations; for examples of expositions we cite the least squares method [5] and the more standard variational method in [9]. These variational methods require that the spaces of piecewise polynomial functions used to approximate the solution of the boundary value problem satisfy certain continuity conditions (imposed on the functions and their derivatives up to a certain order) across the interfaces of adjoining elements. These requirements precipitate rather complicated computations for high order operators.

In the method developed in this work, these complications are entirely avoided. The spaces of functions used to approximate the solution consist of polynomials defined locally on each element with no conditions of continuity across interfaces, and are thus subspaces of $L^{2}$.

The relaxation of all continuity conditions is achieved by working with a special variational formulation of the boundary value problem, which is compatible with the relative arbitrariness of the subspaces. This in essence involves the construction of certain bilinear forms which exploit certain "inverse assumptions" satisfied locally on each element by the approximating functions. Similar considerations allow the result

Received April 15, 1975 ; revised June 24, 1976.

AMS (MOS) subject classifications (1970). Primary 65 N 30. 
that the members of these approximating spaces need not satisfy the prescribed boundary conditions. "Inverse assumptions" of a more global type have been used in nonstandard, conforming variational methods in [7] and by the author [2]

It is proven that the method is optimally convergent in $L^{2}$, and that on each element derivatives of the error up to the order possessed by the solution converge also optimally in $L^{2}$.

Another advantage of the method lies in the fact that, since there are no continuity requirements across the interfaces, arbitrarily high order polynomials may be used on each element, yielding an accuracy of approximation as high as the smoothness of the solution allows. In contrast to methods requiring conforming elements, the use of high order polynomials is restricted by the resulting computational difficulties in meeting these requirements.

It should be noted that although this nonstandard variational method involves a bilinear form which is formally more complicated than the standard variational method, the additional interelement terms are easily computed for example in the case of partitions of the domain into $N$ simplices or $N$-dimensional parallelopipeds.

\section{Notation.}

2.1. Function Spaces. For $D$ a bounded domain in the space $\mathbf{R}^{n}$, of variation $x=\left(x_{1}, x_{2}, \ldots, x_{n}\right), H^{p}(D)$, for real $p$, will denote the Sobolev space of order $p$ of real valued functions on $D .(\cdot, \cdot)_{p, D}$ and $\|\cdot\|_{p, D}$ will denote the inner product and norm, respectively, on $H^{p}(D)$. For definitions and the relevant properties of the spaces $H^{p}(D)$ for $p \geqslant 0$, we refer to [6]. $\bar{D}$ and $\partial D$ will denote the closure and boundary of $D$, respectively.

The space of infinitely differentiable functions on $\bar{D}(D)$ we denote by $C^{\infty}(\bar{D})$ $\left(C^{\infty}(D)\right)$. Following [8] we note that for $p \geqslant 0$, the norm $\|\cdot\|_{-p, D}$ on $H^{-p}(D)$ is defined by

$$
\|v\|_{-p, D}=\sup _{\psi \in C^{\infty}(\bar{D})} \frac{(v, \psi)_{0, D}}{\|\psi\|_{p, D}}, \quad v \in H^{-p}(D) .
$$

Similarly $H^{p}(\partial D)$ will denote the Sobolev space of order $p$ of real valued functions on $\partial D$, with $|\cdot|_{p, \partial D}$ denoting the corresponding norm. Again for definitions and the properties of these spaces we refer to [6]. For brevity, we shall write the inner product and norm on $L^{2}(\partial D)=H^{0}(\partial D)$ as

$$
\langle\varphi, \psi\rangle_{\partial D}=\int_{\partial D} \varphi \psi d \sigma \text { and }|\varphi|_{\partial D}=\{\langle\varphi, \varphi\rangle\}^{1 / 2}
$$

respectively. The space of infinitely differentiable functions on $\partial D$ we denote by $C^{\infty}(\partial D)$.

We shall also use the notation

where

$$
d(D)=\sup _{x, y \in D}|x-y|,
$$

$$
|x|=\left(\sum_{i=1}^{n} x_{i}^{2}\right)^{1 / 2}
$$


2.2. The Boundary Value Problem. Let $\Omega$ be a bounded domain in $\mathbf{R}^{n}$ with boundary $\partial \Omega$ which is assumed to be an $(n-1)$-dimensional manifold of class $C^{\infty}$. We are interested in approximating the solution of the following boundary value problem.

A function $u$ defined on $\bar{\Omega}$ is sought satisfying

$$
\left\{\begin{aligned}
& \Delta^{2} u=f \quad \text { in } \Omega, \\
& u=0 \\
& \frac{\partial u}{\partial n}=0
\end{aligned}\right\} \quad \text { on } \partial \Omega .
$$

The operator $\Delta$ is defined by

$f$ is a given function.

$$
\Delta=\sum_{i=1}^{n} \frac{\partial^{2}}{\partial x_{i}^{2}} \quad \text { and } \quad \Delta^{2} u=\Delta(\Delta u)
$$

Concerning existence, uniqueness and regularity of the solution we have the following theorem.

THEOREM 2.1 (CF. [6]). The mapping $P: C^{\infty}(\Omega) \rightarrow C^{\infty}(\Omega) \times C^{\infty}(\partial \Omega) \times C^{\infty}(\partial \Omega)$ defined by $P u=\left(\Delta^{2} u, u, \partial u / \partial n\right)$ and completed by continuity, is a homeomorphism of $H^{s}(\Omega)$ onto $H^{s-4}(\Omega) \times H^{s-1 / 2}(\partial \Omega) \times H^{s-3 / 2}(\partial \Omega)$ for all $s \geqslant 4$, and there exist constants $\alpha_{s}$ and $\beta_{s}$ such that

$$
\|u\|_{s, \Omega} \leqslant \alpha_{s}\left\{\left\|\Delta^{2} u\right\|_{s-4, \Omega}+|u|_{s-1 / 2, \partial \Omega}+\left|\frac{\partial u}{\partial n}\right|_{s-3 / 2, \partial \Omega}\right\} \leqslant \beta_{s}\|u\|_{s, \Omega} .
$$

Henceforth we make the following assumption on the data $f$ of the boundary value problem (2.1); $f \in H^{s-4}(\Omega)$, for some $s \geqslant 4$. Thus by Theorem 2.1 , the boundary value problem possesses a unique solution $u \in H^{s}(\Omega)$.

2.3. Partitions of $\Omega$. Let $\Pi_{h}(\Omega)=\left\{\Omega_{1}, \Omega_{2}, \ldots, \Omega_{m}\right\}$ be a finite collection of subdomains of $\Omega$, where

$$
h_{i}=d\left(\Omega_{i}\right), \quad i=1,2, \ldots, m,
$$

and

$$
h=\max _{1 \leqslant i \leqslant m} h_{i}
$$

We shall say that $\Pi_{h}(\Omega)$ is of class $G_{\nu}(\Omega)$ if

(i) $\bigcup_{i=1}^{m} \bar{\Omega}_{i}=\bar{\Omega}$;

(ii) $\Omega_{i} \cap \Omega_{j}=\varnothing$ for $i \neq j, i, j=1,2, \ldots, m$;

(iii) Each $\Omega_{i}$ is a regular domain in $\mathbf{R}^{n}$ (i.e., the divergence theorem holds on $\left.\Omega_{i}\right)$, and is starlike, $i=1,2, \ldots, m$.

(iv) The surface $\partial \Omega_{i} \cap \partial \Omega_{j}$ is an $(n-1)$-dimensional manifold which is measurable with respect to Lebesgue measure on $R^{n-1}$ induced by Lebesgue measure on $\mathbf{R}^{n}$.

(v) There exists a constant $0<v<\infty$ such that

$$
\max _{1 \leqslant i \leqslant m} d\left(\bar{S}_{i}\right) \leqslant \nu \min _{1 \leqslant i \leqslant m} d\left(\underline{S}_{i}\right)
$$


where $\bar{S}_{i}$ denotes the circumscribed sphere of $\Omega_{i}$ and $\underline{S}_{i}$ denotes the inscribed sphere of $\Omega_{i}, i=1,2, \ldots, m$.

It follows from (v) that

$$
h \leqslant \nu h_{i}, \quad i=1,2, \ldots, m .
$$

Henceforth, it will be assumed that the domain $\Omega$ is partitioned as $\Pi_{h}(\Omega) \in$ $G_{\nu}(\Omega)$ for some fixed $\nu$ independent of $h_{i}, i=1,2, \ldots, m$. This process is referred to as the divison of $\Omega$ into "regular" finite elements. Note that the condition (iii) above on the elements ensures that $\Pi_{h}(\Omega)$ includes partitions of $\Omega$ into convex elements and more general elements.

With $\Pi_{h}(\Omega)$ we associate the following notation, defining certain important sets. We define the surfaces

$$
\partial \Omega_{i, j}=\partial \Omega_{i} \cap \partial \Omega_{j}, \quad i, j=1,2, \ldots, m .
$$

The orientation of $\partial \Omega_{i, j}$ is defined to be such that the normal points in the direction outwards from the interior of $\Omega_{i}$ at each point.

$$
\begin{aligned}
N_{i} & =\left\{j: \partial \Omega_{i, j} \neq \varnothing\right\}, & i & =1,2, \ldots, m ; \\
\partial \Omega_{i}^{*} & =\partial \Omega_{i} \cap \partial \Omega, & i & =1,2, \ldots, m ;
\end{aligned}
$$

$\partial \Omega_{i}^{*}$ is nonempty in the case where $\Omega_{i}$ and $\Omega$ have portions of their respective bound. aries in common.

The following matrix will be useful; define

$$
\tau_{i, j}=\left\{\begin{array}{ll}
1, & i<j, \\
0, & i \geqslant j,
\end{array} \quad i, j=1,2, \ldots, m .\right.
$$

If $v$ is a function defined on $\Omega$, then $v^{(i)}$ will denote its restriction to $\Omega_{i}, i=1,2$, $\ldots, m$, and we shall denote by $\mathbf{v}$ the corresponding ordered $m$-tuple of functions $\left(v^{(1)}, v^{(2)}, \ldots, v^{(m)}\right)$.

Conversely, for any ordered $m$-tuple of functions $\left(v^{(1)}, v^{(2)}, \ldots, v^{(m)}\right)$ with $v^{(i)}$ measurable on $\Omega_{i}$ we denote by $v$ the function defined a.e. by

$$
v(x)=v^{(i)}(x), \quad x \in \Omega_{i}, i=1,2, \ldots, m .
$$

With each subdomain $\Omega_{i}$ of $\Pi_{h}(\Omega)$ we associate the following boundary operators associated with the boundary value problem $(2.1)$; for $v$ sufficiently smooth on $\bar{\Omega}_{i}$, we define

$$
\begin{array}{ll}
B_{0} v=v, & B_{1} v=\frac{\partial v}{\partial n} \\
S_{0} v=\frac{\partial}{\partial n}(\Delta v), & S_{1} v=-\Delta v .
\end{array}
$$

The operator $S_{k}$ is "naturally" associated with the operator $B_{k}$ prescribed in the boundary value problem (2.1) via the Green's identity for the operator $\Delta^{2}$. For $u, v$ sufficiently smooth on $\bar{\Omega}_{i}$, 


$$
(\Delta u, \Delta v)_{0, \Omega_{i}}=\left(\Delta^{2} u, v\right)_{0, \Omega_{i}}-\sum_{k=0}^{1}\left\langle S_{k} u, B_{k} v\right\rangle_{\partial \Omega_{i}}
$$

Wherever the operators $S_{0}$ and $B_{1}$ appear in the inner products, the normal derivative is taken in the direction in which the normal in the surface is oriented.

The following lemma will be used repeatedly. For an outline of a proof see [3].

Lemma 2.1. There exists a constant $0<C<\infty$ such that for any $\Omega_{i} \in \Pi_{h}(\Omega)$, if $v \in H^{k+1}\left(\Omega_{i}\right), k$ a nonnegative integer, then

$$
\left|\frac{\partial^{k} v}{\partial n^{k}}\right|_{\partial \Omega_{i}}^{2} \leqslant C\left\{\eta^{-1}\|v\|_{k, \Omega_{i}}^{2}+\eta\|v\|_{k+1, \Omega_{i}}^{2}\right\},
$$

for any $\eta>0$. The constant $C$ is independent of $h_{i}, i=1,2, \ldots, m$.

Throughout the rest of the paper, $C$ will denote a generic constant, not necessarily the same in any two places.

3. Finite Dimensional Function Spaces on $\Pi_{h}(\Omega)$. Given $\Pi_{h}(\Omega) \in G_{\nu}(\Omega)$ we assume the existence of function spaces $S_{h_{i}}\left(\Omega_{i}\right), i=1,2, \ldots, m$, consisting of real valued functions defined on $\Omega_{i}$. These spaces of functions are each required to satisfy,

(1) $S_{h_{i}}\left(\Omega_{i}\right)$ is finite dimensional.

(2) $S_{h_{i}}\left(\Omega_{i}\right) \subset H^{l}\left(\Omega_{i}\right)$ for some $l \geqslant 4$.

(3) There exists an integer $r \geqslant l$ and a constant $C$ independent of $h_{i}$, such that for any $v \in H^{s}\left(\Omega_{i}\right), l \leqslant s \leqslant r$,

$$
\inf _{\chi \in S_{h_{i}\left(\Omega_{i}\right)}} \sum_{j=0}^{l} h_{i}^{j}\|v-\chi\|_{j, \Omega_{i}} \leqslant C h_{i}^{s}\|v\|_{s, \Omega_{i}} .
$$

(4) There exists a constant $C$ independent of $h_{i}$ such that for any $\chi \in S_{h_{i}}\left(\Omega_{i}\right)$,

$$
\|\chi\|_{\beta, \Omega_{i}} \leqslant C h_{i}^{-(\beta-\alpha)}\|\chi\|_{\alpha, \Omega_{i}} \text { for all } \alpha \leqslant \beta \leqslant l .
$$

From the assumption (4) and (2.2) it follows that

$$
\|\chi\|_{\beta, \Omega_{i}} \leqslant C h^{-(\beta-\alpha)}\|\chi\|_{\alpha, \Omega_{i}} .
$$

The assumption (4) is referred to as an "inverse assumption".

For example, if $\Pi_{h}(\Omega)$ consists of a triangulation of the domain $\Omega$ (the peripheral triangles will have one "curved" side, i.e., the $\left.\partial \Omega_{i}^{*}\right)$, and we take $S_{h_{i}}\left(\Omega_{i}\right)=P_{r-1}\left(\Omega_{i}\right)$, the set of polynomials of degree $\leqslant r-1$ on $\Omega_{i}$, then all the assumptions (1)-(4) may be verified.

In particular, $S_{h_{i}}\left(\Omega_{i}\right) \subset C^{\infty}\left(\Omega_{i}\right)$,

$$
\inf _{\chi \in P_{r-1}\left(\Omega_{i}\right)} \sum_{j=0}^{s} h^{j}\|v-\chi\|_{j, \Omega_{i}} \leqslant C h^{s}\|v\|_{s, \Omega_{i}}
$$

for all $v \in H^{s}\left(\Omega_{i}\right), s \leqslant r$. See [4] for proofs.

Finally we set

$$
M_{h}=\prod_{i=1}^{m} S_{h_{i}}\left(\Omega_{i}\right)
$$


A generic point of $M_{h}$ shall be denoted by $\chi=\left(\chi^{(1)}, \ldots, \chi^{(m)}\right)$ with $\chi^{(i)} \in S_{h_{i}}\left(\Omega_{i}\right)$, $i=1,2, \ldots, m$.

It is important to observe that the function spaces $S_{h_{i}}\left(\Omega_{i}\right)$ are entirely independent of one another. It is for this reason that the projection method to be defined below, which uses these function spaces to approximate the solution of the boundary value problem, is said to use nonconforming elements. That is there is no conforming condition (continuity or continuity of derivatives) imposed on the functions (say polynomials) as one crosses the interfaces of adjoining elements. Each point of $M_{h}$ may be interpreted as a function in the space $L^{2}(\Omega)$.

The basic idea in the projection method is that if $u$ is the solution of (2.1), then we shall approximate $u$ locally by approximating $u^{(i)}$ by a member of $S_{h_{i}}\left(\Omega_{i}\right), i=$ $1,2, \ldots, m$.

4. The Energy Space. Associated with $\Pi_{h}(\Omega)$ we define a normed linear space $H_{h}$ to be the set contained in the space $\prod_{i=1}^{m} H^{4}\left(\Omega_{i}\right)$, a generic point of which we denote by $\mathbf{v}=\left(v^{(1)}, \ldots, v^{(m)}\right)$, with $v^{(i)} \in H^{4}\left(\Omega_{i}\right)$, furnished with the norm $\|\cdot\|_{H_{h}}$ defined by:

$$
\begin{aligned}
&\|\mathrm{v}\|_{H_{h}}=\left(\sum _ { i = 1 } ^ { m } \left\{\left\|\Delta v^{(i)}\right\|_{0, \Omega_{i}}^{2}\right.\right. \\
&+\sum_{k=0}^{1}\left[\sum _ { j \in N _ { i } } \tau _ { i , j } \left(h^{(2 k-3)}\left|B_{k} v^{(i)}-B_{k} v^{(j)}\right|_{\partial \Omega_{i, j}^{2}}\right.\right. \\
&\left.+h^{-(2 k-3)}\left|S_{k} v^{(i)}\right|_{\partial \Omega_{i, j}}\right) \\
&\left.\left.\left.+h^{(2 k-3)}\left|B_{k} v^{(i)}\right|_{\partial \Omega_{i}^{*}}^{2}+h^{-(2 k-3)}\left|S_{k} v^{(i)}\right|_{\partial \Omega_{i}^{*}}^{2}\right]\right\}\right)^{1 / 2}
\end{aligned}
$$

It is to be understood that the normed linear space $H_{h}$ is not complete; however, if $u \in H^{4}(\Omega)$ is the solution of $(2.1)$, then $\mathbf{u}=\left(u^{(1)}, \ldots, u^{(m)}\right) \in H_{h}$.

Also, clearly, $M_{h}$ is a finite dimensional subspace of $H_{h}$. The following proposition states precisely how points in $H_{h}$ are approximable by points in the subspace $M_{h}$.

Proposition 4.1. There exists a constant $0<C<\infty$ such that if $\mathbf{v} \in H_{h}$ and is such that $v^{(i)} \in H^{s}\left(\Omega_{i}\right), i=1,2, \ldots, m$, for $4 \leqslant s \leqslant r$, then

$$
\inf _{x \in M_{h}}\|\mathrm{v}-\chi\|_{H_{h}} \leqslant C h^{s-2} \sum_{i=1}^{m}\left\|v^{(i)}\right\|_{s, \Omega_{i}} .
$$

where $C$ is independent of $h_{i}, i=1,2, \ldots, m$.

Proof. Let $\mathrm{v} \in H_{h}$. By the property (3) of Section 3 of the spaces $S_{h_{i}}\left(\Omega_{i}\right)$, there exists a $\chi \in M_{h}$ such that

$$
\left\|v^{(i)}-\chi^{(i)}\right\|_{j, \Omega_{i}} \leqslant C h^{s-j}\left\|v^{(i)}\right\|_{s, \Omega_{i}}, \quad i=1,2, \ldots, m,
$$

for all $0 \leqslant j \leqslant l$. 
Set $\mathbf{w}=\mathbf{v}-\chi$. Now from (4.1),

$\|w\|_{H_{h}}^{2} \leqslant \sum_{i=1}^{m}\left\{\left\|w^{(i)}\right\|_{2, \Omega_{i}}\right.$

$$
\begin{aligned}
+\sum_{k=0}^{1}\left[\sum _ { j \in N _ { i } } \tau _ { i , j } \left(2 h^{(2 k-3)}\left|B_{k} w^{(i)}\right|_{\partial \Omega_{i, j}}\right.\right. & +2 h^{(2 k-3)}\left|B_{k} w^{(i)}\right|_{\partial \Omega_{i, j}} \\
& \left.+h^{-(2 k-3)}\left|S_{k} w^{(i)}\right|_{\partial \Omega_{i, j}}^{2}\right) \\
+ & h^{(2 k-3)}\left|B_{k} w^{(i)}\right|_{\partial \Omega_{i}^{*}}^{2}
\end{aligned}
$$

Also by choosing $\eta=h_{i}$ in Lemma 2.1 we have, for $k=0,1$,

$$
\begin{aligned}
\left|B_{k} w^{(i)}\right|_{\partial \Omega_{i}}^{2} & =\left|\frac{\partial^{k} w^{(i)}}{\partial n^{k}}\right|_{\partial \Omega_{i, j}}^{2} \leqslant C\left\{h_{i}^{-1}\left\|w^{(i)}\right\|_{k, \Omega_{i}}^{2}+h_{i}\left\|w^{(i)}\right\|_{k+1, \Omega_{i}}^{2}\right\} \\
& \leqslant C h_{i}^{2(s-k)-1}\left\|v^{(i)}\right\|_{s, \Omega_{i}}^{2} \leqslant C h^{2(s-k)-1}\left\|v^{(i)}\right\|_{s, \Omega_{i}}^{2},
\end{aligned}
$$

where we have used (4.2) and the fact that $2(s-k)-1>0$.

Similarly, for $k=0,1$, we have,

$$
\begin{aligned}
\left|S_{k} w^{(i)}\right|_{\partial \Omega_{i}}^{2} & =\left|\frac{\partial^{1-k}}{\partial n^{1-k}}\left(\Delta w^{(i)}\right)\right|_{\partial \Omega_{i}}^{2} \\
& \leqslant C\left\{h_{i}^{-1}\left\|\Delta w^{(i)}\right\|_{1-k, \Omega_{i}}^{2}+h_{i}\left\|\Delta w^{(i)}\right\|_{2-k, \Omega_{i}}^{2}\right\} \\
& \leqslant C\left\{h_{i}^{-1}\left\|w^{(i)}\right\|_{3-k, \Omega_{i}}^{2}+h_{i}\left\|w^{(i)}\right\|_{4-k, \Omega_{i}}^{2}\right\} \\
& \leqslant C h_{i}^{2(s+k)-7}\left\|v^{(i)}\right\|_{s, \Omega_{i}}^{2} \leqslant C h^{2(s+k)-7}\left\|v^{(i)}\right\|_{s, \Omega_{i}}^{2},
\end{aligned}
$$

again since $2(s+k)-7>0$.

Now using (4.4), (4.5) and (4.2) with $j=2$ to bound the appropriate terms in (4.3) we obtain:

$$
\|\mathrm{w}\|_{H_{h}}^{2} \leqslant C h^{2(s-2)} \sum_{i=1}^{m}\left\|v^{(i)}\right\|_{s, \Omega_{i}}^{2} \leqslant C\left[h^{s-2} \sum_{i=1}^{m}\left\|v^{(i)}\right\|_{s, \Omega_{i}}\right]^{2} .
$$

And so,

$$
\|\mathbf{v}-\chi\|_{H_{h}} \leqslant C h^{s-2} \sum_{i=1}^{m}\left\|v^{(i)}\right\|_{s, \Omega_{i}}
$$

which concludes the proof.

5. A Bilinear Form. We define a bilinear form $B_{h}^{\gamma}(\cdot, \cdot): H_{h} \times H_{h} \rightarrow \mathbf{R}^{1}$ as follows: for $\mathbf{u}=\left(u^{(1)}, \ldots, u^{(m)}\right)$ and $\mathbf{v}=\left(v^{(1)}, \ldots, v^{(m)}\right) \in H_{h}$, 


$$
\begin{aligned}
& B_{h}^{\gamma}(\mathbf{u}, \mathbf{v})=\sum_{i=1}^{m}\left\{\left(\Delta u^{(i)}, \Delta v^{(i)}\right)_{0, \Omega_{i}}\right. \\
& +\sum_{k=0}^{1}\left[\sum _ { j \in N _ { i } } \tau _ { i , j } \left(\left\langle B_{k} u^{(i)}-B_{k} u^{(j)}, S_{k} v^{(i)}\right\rangle_{\partial \Omega_{i, j}}\right.\right. \\
& +\left\langle B_{k} v^{(i)}-B_{k} v^{(j)}, S_{k} u^{(i)}\right\rangle_{\partial \Omega_{i, j}} \\
& \left.+\gamma h^{(2 k-3)}\left\langle B_{k} u^{(i)}-B_{k} u^{(j)}, B_{k} v^{(i)}-B_{k} v^{(j)}\right\rangle_{\partial \Omega_{i, j}}\right) \\
& +\left\langle B_{k} u^{(i)}, S_{k} v^{(i)}\right\rangle_{\partial \Omega_{i}^{*}}+\left\langle B_{k} v^{(i)}, S_{k} u^{(i)}\right\rangle_{\partial \Omega_{i}^{*}} \\
& \left.\left.+\gamma h^{(2 k-3)}\left\langle B_{k} u^{(i)}, B_{k} v^{(i)}\right\rangle_{\partial \Omega_{i}^{*}}\right]\right\} .
\end{aligned}
$$

Here $\gamma$ is a positive constant depending on the constant associated with the inverse assumption (4) of Section 3. Below, it will be shown in Proposition 5.1 that for $\gamma$ chosen sufficiently large, the form $B_{h}^{\gamma}(\cdot, \cdot)$ is positive definite on the subspace $M_{h}$.

Again in (5.1) the surfaces $\partial \Omega_{i, j}$ and $\partial \Omega_{i}^{*}$ are oriented so that the normal points in the direction outwards from the interior of $\Omega_{i}$; wherever the operators $B_{1}$ and $S_{0}$ appear in inner products, the normal derivative on the surface is taken in this direction.

Proposition 5.1. There exists a constant $0<\hat{\gamma}<\infty$ such that if $\gamma \geqslant \hat{\gamma}$, there is a constant $c>0$, independent of $h_{i}, i=1,2, \ldots, m$, such that

$$
B_{h}^{\gamma}(\mathbf{w}, \mathbf{w}) \geqslant c\|\mathrm{w}\|_{H_{h}}^{2} \quad \text { for all } \mathrm{w} \in M_{h} .
$$

Proof. From (5.1), if $\mathrm{w} \in M_{h}$, then

$B_{h}^{\gamma}(\mathrm{w}, \mathrm{w})=\sum_{i=1}^{m}\left\{\left\|\Delta w^{(i)}\right\|_{0, \Omega_{i}}^{2}\right.$

$$
\begin{aligned}
&+\sum_{k=0}^{1}\left[\sum_{j \in N_{i}} \tau_{i, j} 2\left\langle B_{k} w^{(i)}-B_{k} w^{(i)}, S_{k} w^{(i)}\right\rangle_{\partial \Omega_{i, j}}\right. \\
&+\gamma h^{(2 k-3)}\left|B_{k} w^{(i)}-B_{k} w^{(j)}\right|_{\partial \Omega_{i, j}}+2\left\langle B_{k} w^{(i)}, S_{k} w^{(i)}\right\rangle_{\partial \Omega_{i}^{*}} \\
&\left.\left.+\gamma h^{(2 k-3)}\left|B_{k} w^{(i)}\right|_{\partial \Omega_{i}^{*}}^{2}\right]\right\}
\end{aligned}
$$

Now by successive applications of Schwarz' inequality and the arithmetic-geometric mean inequality we obtain

$$
\begin{aligned}
& 2\left\langle B_{k} w^{(i)}, S_{k} w^{(i)}\right\rangle_{\partial \Omega_{i}^{*}} \leqslant 2\left|B_{k} w^{(i)}\right|_{\partial \Omega_{i}^{*}}\left|S_{k} w^{(i)}\right|_{\partial \Omega_{i}^{*}} \\
& =2\left\{\left[\epsilon^{-1} h^{(2 k-3)}\right]^{1 / 2}\left|B_{k} w^{(i)}\right|_{\partial \Omega_{i}^{*}}\right\}\left\{\left[\epsilon^{-1} h^{(2 k-3)}\right]^{-1 / 2}\left|S_{k} w^{(i)}\right|_{\partial \Omega_{i}^{*}}\right\} \\
& \leqslant \frac{h^{(2 k-3)}}{\epsilon}\left|B_{k} w^{(i)}\right|_{\partial \Omega_{i}^{*}}^{2}+\epsilon h^{-(2 k-3)}\left|S_{k} w^{(i)}\right|_{\partial \Omega_{i}^{*}}^{2},
\end{aligned}
$$

for any $\epsilon>0$. 
Similarly

$$
\begin{aligned}
& 2\left\langle B_{k} w^{(i)}-B_{k} w^{(j)}, S_{k} w^{(i)}\right\rangle_{\partial} \Omega_{i, j} \\
& \quad \leqslant \frac{h^{(2 k-3)}}{\epsilon}\left|B_{k} w^{(i)}-B_{k} w^{(j)}\right|_{\partial \Omega_{i, j}}^{2}+\epsilon h^{-(2 k-3)}\left|S_{k} w^{(i)}\right|_{\partial}^{2} \Omega_{i, j},
\end{aligned}
$$

for any $\epsilon>0$.

Using (5.3) and (5.4) in (5.2),

$$
\begin{aligned}
& B_{h}^{\gamma}(\mathrm{w}, \mathrm{w}) \geqslant \sum_{i=1}^{m}\left\{\left\|\Delta w^{(i)}\right\|_{0, \Omega_{i}}^{2}\right. \\
& +\sum_{k=0}^{1}\left[\sum _ { j \in N _ { i } } \tau _ { i , j } \left(\left(\gamma-\frac{1}{\epsilon}\right) h^{(2 k-3)}\left|B_{k} w^{(i)}-B_{k} w^{(i)}\right|_{\partial}^{2} \Omega_{i, j}\right.\right. \\
& \left.-\epsilon h^{-(2 k-3)}\left|S_{k} w^{(i)}\right|_{\partial \Omega_{i, j}}\right)^{2} \\
& \left.\left.+\left(\gamma-\frac{1}{\epsilon}\right) h^{(2 k-3)}\left|B_{k} w^{(i)}\right|_{\partial \Omega_{i}^{*}}^{2}-\epsilon h^{-(2 k-3)}\left|S_{k} w^{(i)}\right|_{\partial}^{2} \Omega_{i}^{*}\right]\right\} \\
& =\sum_{i=1}^{m}\left\{\left\|\Delta w^{(i)}\right\|_{0, \Omega_{i}}^{2}\right. \\
& +\sum_{k=0}^{1}\left[\sum _ { j \in N _ { i } } \tau _ { i , j } \left(\epsilon h^{-(2 k-3)}\left|S_{k} w^{(i)}\right|_{\partial \Omega_{i, j}}^{2}\right.\right. \\
& +\left(\gamma-\frac{1}{\epsilon}\right) h^{(2 k-3)}\left|B_{k} w^{(i)}-B_{k} w^{(j)}\right|_{\partial}^{2} \Omega_{i, j} \\
& \left.-2 \epsilon h^{-(2 k-3)}\left|S_{k} w^{(i)}\right|_{\partial \Omega_{i, j}}\right) \\
& +\epsilon h^{-(2 k-3)}\left|S_{k} w^{(i)}\right|_{\partial \Omega_{i}^{*}}^{2}+\left(\gamma-\frac{1}{\epsilon}\right) h^{(2 k-3)}\left|B_{k} w^{(i)}\right|_{\partial \Omega_{i}^{*}}^{2} \\
& \left.\left.-2 \epsilon h^{-(2 k-3)}\left|S_{k} w^{(i)}\right|_{\partial}^{2} \Omega_{i}^{*}\right]\right\} \text {. }
\end{aligned}
$$

Now by Lemma 2.1 , with $\eta=h_{i}, k=0,1$,

$$
\begin{aligned}
\left|S_{k} w^{(i)}\right|_{\partial \Omega_{i}}^{2} & =\left|\frac{\partial^{1-k}}{\partial \eta^{1-k}}\left(\Delta w^{(i)}\right)\right|_{\partial \Omega_{i}}^{2} \\
& \leqslant C\left\{h_{i}^{-1}\left\|\Delta w^{(i)}\right\|_{1-k, \Omega_{i}}^{2}+h_{i}\left\|\Delta w^{(i)}\right\|_{2-k, \Omega_{i}}^{2}\right\} .
\end{aligned}
$$

Since $\Delta w^{(i)} \in S_{h_{i}}\left(\Omega_{i}\right)$, we may apply the inverse assumption (4) to obtain from (5.6),

$$
\begin{aligned}
\left|S_{k} w^{(i)}\right|_{\partial \Omega_{i}}^{2} & \leqslant C\left\{h_{i}^{-1} h_{i}^{-2(1-k)}\left\|\Delta w^{(i)}\right\|_{0, \Omega_{i}}^{2}+h_{i} h_{i}^{-2(2-k)}\left\|\Delta w^{(i)}\right\|_{0, \Omega_{i}}^{2}\right\} \\
& =C h_{i}^{(2 k-3)}\left\|\Delta w^{(i)}\right\|_{0, \Omega_{i}}^{2}, \quad k=0,1 .
\end{aligned}
$$


Hence from (5.7) and (2.2), since $2 k-3<0$,

$$
h^{-(2 k-3)}\left|S_{k} w^{(i)}\right|_{\partial \Omega_{i}}^{2} \leqslant C \nu^{3-2 k}\left\|\Delta w^{(i)}\right\|_{0, \Omega_{i}}^{2} \leqslant C\left\|\Delta w^{(i)}\right\|_{0, \Omega_{i}}^{2}
$$

Using the bounds (5.8) in (5.5), we arrive at

$$
B_{h}^{\gamma}(\mathrm{w}, \mathrm{w}) \geqslant \sum_{i=1}^{m}\left\{(1-\epsilon C)\left\|\Delta w^{(i)}\right\|_{0, \Omega_{i}}^{2}\right.
$$

$$
\begin{aligned}
+\sum_{k=0}^{1}\left[\sum _ { j \in N _ { i } } \tau _ { i , j } \left(\left(\gamma-\frac{1}{\epsilon}\right) h^{(2 k-3)}\left|B_{k} w^{(i)}-B_{k} w^{(j)}\right|_{\partial \Omega_{i, j}^{2}}\right.\right. \\
\left.+\epsilon h^{-(2 k-3)}\left|S_{k} w^{(i)}\right|_{\partial \Omega_{i, j}}^{2}\right) \\
\left.\left.+\left(\gamma-\frac{1}{\epsilon}\right) h^{(2 k-3)}\left|B_{k} w^{(i)}\right|_{\partial \Omega_{i}^{*}}^{2}+\epsilon h^{-(2 k-3)}\left|S_{k} w^{(i)}\right|_{\partial \Omega_{i}^{*}}^{2}\right]\right\} .
\end{aligned}
$$

In (5.9), $\epsilon>0$ has been so far unspecified. We now choose $\hat{\epsilon}$ such that $1-$ $\hat{\epsilon} C>0$, and $\hat{\gamma}$ such that $\hat{\gamma}-\hat{\epsilon}^{-1}>0$. Then for $\gamma \geqslant \hat{\gamma}$ we have from (5.9)

$$
B_{h}^{\gamma}(\mathbf{w}, \mathbf{w}) \geqslant \hat{C}\|\mathbf{w}\|_{H_{h}}^{2}
$$

where $\hat{C}=\min \left\{(1-\hat{\epsilon} C),\left(\hat{\gamma}-\hat{\epsilon}^{-1}\right), \hat{\epsilon}\right\}$. This concludes the proof.

Proposition 5.2. There exists a constant $0<C<\infty$ such that

$$
\left|B_{h}^{\gamma}(\mathbf{u}, \mathbf{v})\right| \leqslant C\|\mathbf{u}\|_{H_{h}}\|\mathbf{v}\|_{H_{h}},
$$

for all $\mathbf{u}, \mathbf{v} \in H_{h} . \quad C$ is independent of $h_{i}, i=1,2, \ldots, m$.

Proof. Let $\mathbf{u}, \mathbf{v} \in H_{h}$; then applying Schwarz' inequality to each term of

$$
\begin{aligned}
\left|B_{h}^{\gamma}(\mathbf{u}, \mathbf{v})\right| \leqslant \sum_{i=1}^{m}\{ & \left\|\Delta u^{(i)}\right\|_{0, \Omega_{i}}\left\|\Delta v^{(i)}\right\|_{0, \Omega_{i}} \\
& +\sum_{k=0}^{1}\left[\sum _ { j \in N _ { i } } \tau _ { i , j } \left(h^{\left(\frac{2 k-3}{2}\right)}\left|B_{k} u^{(i)}-B_{k} u^{(j)}\right|_{\partial \Omega_{i, j}} h^{-\left(\frac{2 k-3}{2}\right)}\left|S_{k} v^{(i)}\right|_{\partial \Omega_{i, j}}\right.\right.
\end{aligned}
$$

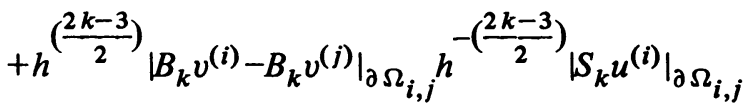

$$
\begin{aligned}
& \left.+\gamma h^{(2 k-3)}\left|B_{k} u^{(i)}-B_{k} u^{(j)}\right|_{\partial \Omega_{i, j}}\left|B_{k} v^{(i)}-B_{k} v^{(j)}\right|_{\partial \Omega_{i, j}}\right) \\
& +h^{\left(\frac{2 k-3}{2}\right)}\left|B_{k} u^{(i)}\right|_{\partial \Omega_{i}^{*}} h^{-\left(\frac{2 k-3}{2}\right)}\left|S_{k} v^{(i)}\right|_{\partial \Omega_{i}^{*}} \\
& +h^{\left(\frac{2 k-3}{2}\right)}\left|B_{k} v^{(i)}\right|_{\partial \Omega_{i}^{*}} h^{-\left(\frac{2 k-3}{2}\right)}\left|S_{k} u^{(i)}\right|_{\partial \Omega_{i}^{*}}
\end{aligned}
$$

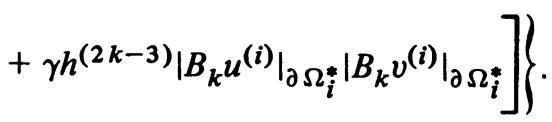


Again by Schwarz' inequality, applied to (5.10),

$$
\begin{aligned}
& B_{h}^{\gamma}(\mathbf{u}, \mathbf{v}) \leqslant\left(\sum _ { i = 1 } ^ { m } \left\{\left\|\Delta u^{(i)}\right\|_{0, \Omega_{i}}^{2}\right.\right. \\
& +\sum_{k=0}^{1}\left[\sum _ { j \in N _ { i } } \tau _ { i , j } \left((1+\gamma) h^{(2 k-3)}\left|B_{k} u^{(i)}-B_{k} u^{(j)}\right|_{\partial}^{2} \Omega_{i, j}\right.\right. \\
& \left.+h^{-(2 k-3)}\left|S_{k} u^{(i)}\right|_{\partial \Omega_{i, j}^{2}}\right) \\
& \left.\left.\left.+(1+\gamma) h^{(2 k-3)}\left|B_{k} u^{(i)}\right|_{\partial \Omega_{i}^{*}}^{2}+h^{-(2 k-3)}\left|S_{k} u^{(i)}\right|_{\partial}^{2} \Omega_{i}^{*}\right]\right\}\right) \\
& \times\left(\sum _ { i = 1 } ^ { m } \left\{\left\|\Delta v^{(i)}\right\|_{0, \Omega_{i}}^{2}\right.\right. \\
& +\sum_{k=0}^{1}\left[\sum _ { j \in N _ { i } } \tau _ { i , j } \left((1+\gamma) h^{(2 k-3)}\left|B_{k} v^{(i)}-B_{k} v^{(j)}\right|_{\partial \Omega_{i, j}}\right.\right. \\
& \left.+h^{-(2 k-3)}\left|S_{k} v^{(i)}\right|_{\partial}^{2} \Omega_{i, j}\right) \\
& \left.\left.\left.+(1+\gamma) h^{(2 k-3)}\left|B_{k} v^{(i)}\right|_{\partial \Omega_{i}^{*}}^{2}+h^{-(2 k-3)}\left|S_{k} v^{(i)}\right|_{\partial \Omega_{i}^{*}}^{2}\right]\right\}\right) \\
& \leqslant(1+\gamma)\|\mathbf{u}\|_{H_{h}}\|\mathbf{v}\|_{H_{h}},
\end{aligned}
$$

which gives the result and concludes the proof.

We now show how the bilinear form $B_{h}^{\gamma}(\cdot, \cdot)$ characterizes the solution of the boundary value problem (2.1) via a variational equation on the space $H_{h}$.

If $\mathbf{u}, \mathbf{v} \in H_{h}$, then an application of Green's identity to each subdomain $\Omega_{i}$ yields from (2.3),

$$
\begin{aligned}
& \sum_{i=1}^{m}\left(\Delta u^{(i)}, \Delta v^{(i)}\right)_{0, \Omega_{i}} \\
& =\sum_{i=1}^{m}\left\{\left(\Delta^{2} u^{(i)}, v^{(i)}\right)_{0, \Omega_{i}}-\sum_{k=0}^{1}\left\langle S_{k} u^{(i)}, B_{k} v^{(i)}\right\rangle_{\partial \Omega_{i}}\right\} \\
& =\sum_{i=1}^{m}\left\{\left(\Delta^{2} u^{(i)}, v^{(i)}\right)_{0, \Omega_{i}}\right. \\
& -\sum_{k=0}^{1}\left[\sum_{j \in N_{i}} \tau_{i, j}\left(\left\langle S_{k} u^{(i)}, B_{k} v^{(i)}\right\rangle_{\partial \Omega_{i, j}}-\left\langle S_{k} u^{(j)}, B_{k} v^{(j)}\right\rangle_{\partial \Omega_{i, j}}\right)\right. \\
& \left.\left.+\left\langle S_{k} u^{(i)}, B_{k} v^{(i)}\right\rangle_{\partial \Omega_{i}^{*}}\right]\right\} \text {. }
\end{aligned}
$$

Substituting (5.11) into (5.1), we obtain 
$B_{h}^{\gamma}(\mathrm{u}, \mathrm{v})=\sum_{i=1}^{m}\left\{\left(\Delta^{2} u^{(i)}, v^{(i)}\right)_{0, \Omega_{i}}\right.$

$$
+\sum_{k=0}^{1}\left[\sum _ { j \in N _ { i } } \tau _ { i , j } \left(\left\langle B_{k} u^{(i)}-B_{k} u^{(j)}, S_{k} v^{(i)}\right\rangle_{\partial \Omega_{i, j}}\right.\right.
$$

$$
\begin{array}{r}
+\gamma h^{(2 k-3)}\left\langle B_{k} u^{(i)}-B_{k} u^{(j)}, B_{k} v^{(i)}-B_{k} v^{(j)}\right\rangle_{\partial \Omega_{i, j}} \\
\left.-\left\langle S_{k} u^{(i)}-S_{k} u^{(j)}, B_{k} v^{(j)}\right\rangle_{\partial \Omega_{i, j}}\right) \\
\left.\left.+\left\langle B_{k} u^{(i)},\left(S_{k}+\gamma h^{(2 k-3)} B_{k}\right) v^{(i)}\right\rangle_{\partial \Omega_{i}^{*}}\right]\right\} .
\end{array}
$$

Hence if $u \in H^{4}(\Omega)$ is the solution of (2.1), from (5.12),

$$
B_{h}^{\gamma}(\mathbf{u}, \mathbf{v})=(f, v)_{0, \Omega} \quad \text { for all } \mathbf{v} \in H_{h} .
$$

We remark that (5.13) is the variational equation satisfied by $\mathbf{u}$, the solution of (2.1). $B_{h}^{\gamma}(\mathbf{u}, \mathbf{v})$ is explicitly computable in terms of the data, $f$, of the boundary value problem, for each $\mathbf{v} \in H_{h}$.

6. The Finite Element Approximation and Error Estimates. We now prove the essential results of the paper. First, we establish the existence and uniqueness of a $u_{h} \in M_{h}$ the finite element approximation to $u$, the solution of (2.1). It will be seen that $u_{h}$ is explicitly computable using the data of the boundary value problem, by solving a certain system of linear algebraic equations.

Finally, we derive quasi-optimal error estimates in the Sobolev spaces $H^{-p}(\Omega)$, $0 \leqslant p \leqslant r-4$ (in particular in $L^{2}(\Omega)$ ), and show in addition that the higher order derivatives of $u_{h}$ defined locally on each $\Omega_{i}$ converge in $L^{2}\left(\Omega_{i}\right)$ to those of $u$, again at an optimal rate.

THEOREM 6.1. Let $u$ be the solution of the boundary value problem (2.1), with $u \in H^{s}(\Omega)$ for some $s \geqslant 4$. Then for each $\Pi_{h}(\Omega)$ and corresponding $M_{h}$, there exists a unique $\mathrm{u}_{h} \in M_{h}$ satisfying

$$
B_{h}^{\gamma}\left(\mathbf{u}_{h}, \mathbf{v}\right)=(f, v)_{0, \Omega} \quad \text { for all } v \in M_{h} .
$$

Furthermore, there exists a constant $c$ independent of $h_{i}, i=1,2, \ldots, m$, such that

$$
\begin{gathered}
\left\|\mathrm{u}-\mathrm{u}_{h}\right\|_{H_{h}} \leqslant C h^{s-2}\|u\|_{s, \Omega}, \\
\left\|u-u_{h}\right\|_{-p, \Omega} \leqslant C h^{s+p}\|u\|_{s, \Omega} \quad \text { for } 0 \leqslant p \leqslant r-4, \\
\sum_{i=1}^{m}\left\|D^{\alpha}\left(u^{(i)}-u_{h}^{(i)}\right)\right\|_{0, \Omega_{i}} \leqslant C h^{s-|\alpha|}\|u\|_{s, \Omega_{i}}
\end{gathered}
$$

for $0 \leqslant|\alpha| \leqslant s, l \leqslant s \leqslant r$.

Proof. Solving Eq. (6.1) for $\mathbf{u}_{h} \in M_{h}$ is equivalent to solving a system of $\sigma(m)$ $\times \sigma(m)$ linear algebraic equations for the coefficients of $\mathbf{u}_{h}$ with respect to a basis of $M_{h}$, where $\sigma(m)$ is the dimension of $M_{h}$. 
Since by Proposition 5.1 the form $B_{h}^{\gamma}(\cdot, \cdot)$ is positive definite on $M_{h}$ it follows that the matrix is itself positive definite and so nonsingular. Hence $\mathbf{u}_{h}$ exists uniquely.

To derive (6.2) we first note that by Proposition 4.1 there exists a $\xi \in M_{h}$ such that

$$
\|\mathbf{u}-\xi\|_{H_{h}} \leqslant C h^{s-2}\|u\|_{s, \Omega} .
$$

Now since $\xi-u_{h} \in M_{h}$, by Proposition 5.1,

$$
\begin{aligned}
\left\|\xi-u_{h}\right\|_{H_{h}}^{2} & \leqslant C B_{h}^{\gamma}\left(\xi-u_{h}, \xi-u_{h}\right)=C B_{h}^{\gamma}\left(\xi-u+u-u_{h}, \xi-u_{h}\right) \\
& =C B_{h}^{\gamma}\left(\xi-u, \xi-u_{h}\right)+C B_{h}^{\gamma}\left(u-u_{h}, \xi-u_{h}\right) .
\end{aligned}
$$

Now from (6.1) and (5.13) it follows that

$$
B_{h}^{\gamma}\left(\mathbf{u}-\mathbf{u}_{h}, \chi\right)=0 \text { for all } \chi \in M_{h} .
$$

Hence (6.6) reduces to

$$
\left\|\xi-u_{h}\right\|_{H_{h}}^{2} \leqslant C B_{h}^{\gamma}\left(\xi-\mathbf{u}, \xi-u_{h}\right) \leqslant C\|\xi-\mathbf{u}\|_{H_{h}}\left\|\xi-u_{h}\right\|_{H_{h}},
$$

where we have used Proposition 5.2.

Hence from (6.8) and (6.5)

$$
\left\|\xi-u_{h}\right\|_{H_{h}} \leqslant C\|\xi-u\|_{H_{h}} \leqslant C h^{s-2}\|u\|_{s, \Omega} .
$$

Finally combining this last estimate with (6.5),

$$
\left\|\mathbf{u}-\mathbf{u}_{h}\right\|_{H_{h}} \leqslant\|\mathbf{u}-\xi\|_{H_{h}}+\left\|\xi-u_{h}\right\|_{H_{h}} \leqslant C h^{s-2}\|u\|_{s, \Omega},
$$

which is the required result.

We now derive (6.3). Set $\mathrm{e}=\mathrm{u}-\mathrm{u}_{h}$, and let $\psi \in C^{\infty}(\bar{\Omega})$. By Theorem 2.1 there exists a unique $\varphi \in C^{\infty}(\Omega)$ such that

$$
\begin{aligned}
& \Delta^{2} \varphi=\psi \quad \text { in } \Omega, \\
& \left.\begin{array}{r}
\varphi=0 \\
\partial \varphi / \partial n=0
\end{array}\right\} \text { on } \partial \Omega
\end{aligned}
$$

and for all $t \geqslant 4$,

$$
\|\varphi\|_{t, \Omega} \leqslant \alpha_{t}\|\psi\|_{t-4, \Omega}
$$

Also by Proposition 4.1 there exists a $\zeta \in M_{h}$ such that

$$
\|\varphi-\zeta\|_{H_{h}} \leqslant C h^{t-2}\|\varphi\|_{t, \Omega}
$$

From (5.12) we have

$$
B_{h}^{\gamma}(\varphi, \mathrm{e})=(\psi, e)_{0, \Omega} .
$$

Hence from this last equation, (6.7), Proposition 5.2, (6.9) and (6.10) we have 


$$
\begin{aligned}
(\psi, e)_{0, \Omega} & =B_{h}^{\gamma}(\varphi, e)=B_{h}^{\gamma}(\mathrm{e}, \varphi) \\
& =B_{h}^{\gamma}(\mathrm{e}, \varphi-\zeta) \leqslant C\|e\|_{H_{h}}\|\varphi-\zeta\|_{H_{h}} \leqslant C h^{t-2}\|\varphi\|_{t, \Omega}\|e\|_{H_{h}} \\
& \leqslant C h^{t-2}\|\psi\|_{t-4, \Omega}\|e\|_{H_{h}}, \quad 4 \leqslant t \leqslant r
\end{aligned}
$$

on $(\psi, e)_{0, \Omega} /\|\psi\|_{t-4, \Omega} \leqslant C h^{t-2}\|e\|_{H_{h}}$.

Hence

$$
\|e\|_{-(t-4), \Omega} \leqslant C h^{t-2}\|e\|_{H_{h}}
$$

setting $p=t-4$, and using the estimate (6.2),

$$
\|e\|_{-p, \Omega} \leqslant C h^{p+2}\|e\|_{H_{h}} \leqslant C h^{s+p}\|u\|_{s, \Omega}
$$

for $0 \leqslant p \leqslant r-4, l \leqslant s \leqslant r$, which is (6.3).

To derive (6.4), let $\xi$ be chosen as in (6.5). Then in particular,

$$
\left\|u^{(i)}-\xi^{(i)}\right\|_{j, \Omega_{i}} \leqslant C h^{s-j}\left\|u^{(i)}\right\|_{s, \Omega_{i}} \text { for all } 0 \leqslant j \leqslant l .
$$

Hence for $0 \leqslant q \leqslant s$, using (6.11),

$$
\begin{aligned}
\sum_{i=1}^{m}\left\|e^{(i)}\right\|_{q, \Omega_{i}} & \leqslant \sum_{i=1}^{m}\left\{\left\|u^{(i)}-\xi^{(i)}\right\|_{q, \Omega_{i}}+\left\|\xi^{(i)}-u_{h}^{(i)}\right\|_{q, \Omega_{i}}\right\} \\
& \leqslant C h^{s-q} \sum_{i=1}^{m}\left\|u^{(i)}\right\|_{s, \Omega_{i}}+\sum_{i=1}^{m}\left\|\xi^{(i)}-u_{h}^{(i)}\right\|_{q, \Omega_{i}}
\end{aligned}
$$

Now using the inverse assumption (4) on each $\Omega_{i}$, (6.12) becomes

$$
\begin{aligned}
\sum_{i=1}^{m}\left\|e^{(i)}\right\|_{q, \Omega_{i}} & \leqslant C h^{s-q}\|u\|_{s, \Omega}+C \sum_{i=1}^{m} h_{i}^{-q}\left\|\xi^{(i)}-u_{h}^{(i)}\right\|_{0, \Omega_{i}} \\
& \leqslant C h^{s-q}\|u\|_{s, \Omega}+C h^{-q} \nu^{q} \sum_{i=1}^{m}\left\|\xi^{(i)}-u_{h}^{(i)}\right\|_{0, \Omega_{i}} \\
& \leqslant C h^{s-q}\|u\|_{s, \Omega}+C h^{-q}\left\|\xi-u_{h}\right\|_{0, \Omega} \\
& \leqslant C h^{s-q}\|u\|_{s, \Omega}+C h^{-q}\left\{\|\xi-u\|_{0, \Omega}+\left\|u-u_{h}\right\|_{0, \Omega}\right\} .
\end{aligned}
$$

Finally from (6.3) with $p=0$ and (6.11),

$$
\sum_{i=1}^{m}\left\|e^{(i)}\right\|_{q, \Omega_{i}} \leqslant C h^{s-q}\|u\|_{s, \Omega}
$$

This gives (6.4) and concludes the proof.

Division of Engineering and Applied Physics

Harvard University

Cambridge, Massachusetts 02138

1. I. BABUŠKA \& M. ZLÁMAL, "Nonconforming elements in the finite element method with penalty," SIAM J. Numer. Anal., v. 10, 1973, pp. 863-875. MR 49 \# 10168.

2. G. BAKER, Projection Methods for Boundary Value Problems for Elliptic and Parabolic Equations with Discontinuous Coefficients, Ph. D. Thesis, Cornell Univ., 1973. 
3. J. H. BRAMBLE, T. DUPONT \& V. THOMÉE, "Projection methods for Dirichlet's problem in approximating polygonal domains with boundary-value corrections," Math. Comp., v. 26, 1972, pp. 869-879. MR 49 \# 8397.

4. J. H. BRAMBLE \& S. R. HILBERT, "Estimation of linear functionals on Sobolev spaces with application to Fourier transforms and spline interpolation," SIAM J. Numer. Anal., v. 7, 1970, pp. 112-124. MR 41 \# 7819.

5. J. H. BRAMBLE \& A. H. SCHATZ, "Least squares methods for $2 m$ th order elliptic boundary-value problems," Math. Comp., v. 25, 1971, pp. 1-32. MR 45 \# 4657.

6. J. L. LIONS \& E. MAGENES, Problèmes aux Limites Non Homogènes et Applications, Vols. 1, 2, Dunod, Paris, 1968. MR 40 \# 512, \# 513.

7. J. A. NITSCHE, "Über ein Variationsprinzip zur Lösung von Dirichlet-Problemen bei Verwendung von Teilräumen, die keinen Randbedingungen unterworfen sind," Abh. Math. Sem. Univ. Hamburg, v. 36, 1971 , pp. 9-15. MR 49 \# 6649.

8. M. SCHECHTER, “On $L^{p}$ estimates and regularity. II," Math. Scand., v. 13, 1963, pp. 47-69. MR 32 \# 6052.

9. G. STRANG, "The finite element method and approximation theory," in Numerical Solution of Partial Differential Equations, II (SYNSPADE 1970), (Proc. Sympos., Univ. of Maryland, 1970), edited by B. E. Hubbard, Academic Press, New York, 1971, pp. 547-583. MR 44 \# 4926. 